\title{
Estratégias de cuidado à saúde desenvolvidas pela pessoa idosa em sua vivência com o HIV/AIDS
}

\author{
Health care strategies developed by the elderly in their experience with HIV/AIDS \\ Estrategias de atención de la salud desarrolladas por personas mayores en su experiencia con el \\ VIH/SIDA
}

Recebido: 01/12/2021 | Revisado: 10/12/2021 | Aceito: 11/12/2021 | Publicado: 20/12/2021

\author{
Luciária Silva Souza \\ ORCID: https://orcid.org/0000-0002-8479-237X \\ Faculdade Independente do Nordeste, Brasil \\ E-mail: luciariasilvasouza@gmail.com \\ Naiane Pereira dos Santos \\ ORCID: https://orcid.org/0000-0001-7440-5316 \\ Faculdade Independente do Nordeste, Brasil \\ E-mail: santosnayane.n@ hotmail.com \\ Luciana Araújo dos Reis \\ ORCID: https://orcid.org/0000-0001-8416-7104 \\ Universidade Estadual do Sudoeste da Bahia, Brasil \\ E-mail: luanareis@ fainor.com.br \\ Alessandra Souza de Oliveira \\ ORCID: https://orcid.org/0000-0002-4459-1493 \\ Universidade Estadual do Sudoeste da Bahia, Brasil \\ E-mail: bahiale23@yahoo.com.br \\ Luana Araújo dos Reis \\ ORCID: https://orcid.org/0000-0002-9263-083X \\ Faculdade Independente do Nordeste, Brasil \\ E-mail: luanareis@fainor.com.br
}

\begin{abstract}
Resumo
Tem-se evidenciado nos últimos anos um crescimento significativo de idosos vivendo com HIV/AIDS, nesse sentido, o desenvolvimento de estratégias multidisciplinares contribuíram para a garantia da sobrevida e das condições de vida dessas pessoas. O presente estudo tem como objetivo conhecer as estratégias de cuidado à saúde desenvolvidas pela pessoa idosa em sua vivência com o HIV/AIDS. Trata-se de um estudo exploratório, de caráter descritivo e natureza quanti-qualitativa, desenvolvida em um Centro de Atenção e Apoio especializado de referência em atendimento para pessoas que convivem com IST's e HIV/AIDS em um município baiano. A amostra constituiu-se de 26 pessoas com idade igual ou superior a 60 anos diagnosticadas com HIV/AIDS e que fazem tratamento no centro de referência. Como instrumento para coleta de dados foi utilizado o Mini-Exame do Estado Mental/MEEM, questionário com dados sociodemográficos e roteiro semiestruturado para entrevista. Os dados qualitativos serão analisados e interpretados através do conteúdo proposto por Bardin. Os resultados demonstraram que as estratégias de enfrentamento desenvolvidas pelos idosos soropositivos foram a adesão ao tratamento TARV, religiosidade, prevenção sexual, prática de exercício físico e alimentação, além de obter apoio institucional dos profissionais de saúde e da rede familiar. Conclui-se que as estratégias utilizadas pelos idosos para o enfrentamento da doença constitui importante ferramenta de fortalecimento para aumento da longevidade e incentiva como enfrentar positivamente a condição sorológica.
\end{abstract}

Palavras-chave: Idoso; HIV/AIDS; Estratégia; Cuidado.

\begin{abstract}
In recent years, there has been a significant increase in the number of elderly people living with HIV/AIDS. In this sense, the development of multidisciplinary strategies has contributed to guaranteeing the survival and living conditions of these people. This study aims to understand the health care strategies developed by the elderly in their experience with HIV/AIDS. This is an exploratory study, with a descriptive character and quanti-qualitative nature, developed in a specialized Care and Support Center of reference in care for people living with STIs and HIV/AIDS in a municipality in Bahia. The sample consisted of 26 people aged over 60 years diagnosed with HIV/AIDS and undergoing treatment at the referral center. As an instrument for data collection, the Mini-Mental State Examination/MMSE, a questionnaire with sociodemographic data and a semi-structured interview script were used. Qualitative data Will be analyzed and interpreted through the content proposed by Bardin. The results showed that the
\end{abstract}


coping strategies developed by the HIV-positive elderly were adherence to ART, religiosity, sexual prevention, physical exercise and nutrition, in addition to obtaining institutional support from health Professionals and the family network. It is concluded that the strategies used by the elderly to cope with the disease constitute an important strengthening tool to increase longevity and encourage how to face the serological condition positively.

Keywords: Elderly; HIV/AIDS; Strategy; Caution.

\section{Resumen}

En los últimos años se ha producido un aumento significativo en el número de personas mayores que viven con el VIH / SIDA, en este sentido, el desarrollo de estrategias multidisciplinarias ha contribuido a garantizar la supervivencia y las condiciones de vida de estas personas. Este estudio tiene como objetivo comprender las estrategias de atención de la salud desarrolladas por las personas mayores em su experiencia con el VIH / SIDA. Se trata de un estudio exploratorio, de carácter descriptivo y cuanti-cualitativo, desarrollado em un Centro de Atención y Soporte Especializado de referencia en atención a personas viviendo con ITS y VIH / SIDA en un municipio de Bahía. La muestra estuvo formada por 26 personas mayores de 60 años diagnosticadas de VIH / SIDA en tratamiento en el centro de referencia. Como instrumento de recogida de datos se utilizó el Mini Examen del Estado Mental / MMSE, un cuestionario con datos sociodemográficos y un guion de entrevista semiestructurada. Los datos cualitativos serán analizados e interpretados a través del contenido propuesto por Bardin. Los resultados mostraron que las estrategias de afrontamiento desarrolladas por los ancianos VIH positivos fueron la adherencia al TAR, la religiosidad, la prevención sexual, el ejercicio físico y la nutrición, además de obtener el apoyo institucional de los profesionales de la salud y la red familiar. Se concluye que las estrategias que utilizan los ancianos para afrontar la enfermedad constituyen una importante herramienta de fortalecimiento para incrementar la longevidad y fomentar cómo afrontar positivamente la condición serológica.

Palabras clave: Anciano; VIH/SIDA; Estrategia; Precaución.

\section{Introdução}

Tem-se evidenciado nos últimos anos um aumento gradativo do envelhecimento populacional em todo mundo. Tal fator está correlacionado aos aspectos de melhorias nas políticas de saúde, na evolução da medicina e da tecnologia moderna que permite uma melhoria na qualidade de vida (Bastos et al., 2018). Além disso, há uma diminuição da taxa de fecundidade e de mortalidade, como também uma extensão da expectativa de vida, a qual tem possibilitado uma transição sociodemográfica e a longevidade da população (Nardelli et al., 2017; Bittencourt et al., 2015).

Relativo a este contexto, nota-se um crescimento significativo de idosos vivendo com o Vírus da Imunodeficiência Humana (HIV) e da Síndrome da Imunodeficiência Adquirida (AIDS). A mudança no comportamento sexual dos idosos vem sendo apontada como uma das justificativas para a exposição de pessoas desta faixa etária aos riscos de acometimento por Infecções Sexualmente Transmissíveis (IST's). Uma vez que, aderiram ao uso de fármacos para tratamento hormonal e de disfunção erétil, os quais permitem o estabelecimento de novas posturas que oportunizam o prolongamento e o aperfeiçoamento de uma vida sexual ativa, permitindo a descobertas de novas experiências (Bastos et al., 2018).

Anteposto, o HIV é um retrovírus da subfamília Lentiviridade, que tem como característica principal a capacidade de se ligar aos receptores e adentrar às células de defesa, conhecida como desencadeadores do sistema inume, chamados de linfócitos T CD4+, podendo assim, ser capaz de comprometer o sistema imunológico. Quando há proliferação do vírus no organismo e uma imunossupressão profunda da pessoa infectada, é estabelecido um quadro denominado de AIDS (Pedrosa; Lebrego, 2016; Affeldt et al., 2015).

Embora a epidemia da AIDS tenha ocorrido em 1980, a sociedade de maneira geral, ainda carrega uma bagagem de preconceito e mitos, arraigada ao estigma e exclusão social das pessoas que convivem com a doença (Silva; Loreto; Mafra, 2017). Nesse sentido, diante da perspectiva social, a maioria da população acredita que não existe o exercício da prática sexual entre pessoas idosas e os considera como assexuais (Bittencourt et al., 2015). Outro requisito importante a ser descrito é que normalmente os idosos não possuem a prática de fazer uso do preservativo. Isso em decorrência de confiarem em seus companheiros ou por não estarem em uma fase reprodutiva ou também por terem medo de haver uma redução da ereção (Sousa, 2019). Ademais, entendem que não são suscetíveis a infecção de HIV/AIDS, uma vez que, há uma visão estereotipada 
que IST's acometem somente indivíduos jovens. Assim, esses aspectos podem acarretar impasses para prevenção, como também ser um contribuinte para que os idosos sejam passíveis de uma maior exposição ao risco de infecção (Aguiar; Leal; Marques, 2020).

Atrelado a isso, ainda há a questão do diagnóstico tardio entre os idosos. Essa problemática se dá devido aos idosos não serem reconhecidos como grupos vulneráveis à infecção do HIV/AIDS pelos profissionais de saúde, além dos próprios idosos não enxergarem como pessoas suscetíveis às IST's. Por conseguinte, os profissionais de saúde associam os primeiros sinais e sintomas característicos de infecções oportunistas que acontecem na AIDS a outras patologias mais comuns nessa faixa etária. Por vezes, o teste de HIV/AIDS não é disponibilizado com frequência para os idosos, fator que aumenta a possibilidade de serem diagnosticados tardiamente, contribuindo assim para uma internação hospitalar. Isto posto, é possível notar que muitos diagnósticos acontecem no nível secundário de saúde e em alguns casos nos serviços terciários, ao invés de acontecer no nível primário seguindo os pressupostos da hierarquia dos serviços de saúde (Alencar \& Ciosak, 2015).

À vista disso, é possível afirmar que a sexualidade do idoso é negligenciada pela sociedade e por órgãos públicos. Posto que, não são desenvolvidas estratégias de promoção e prevenção contra IST's para este grupo etário e tais ações são consideradas influenciadoras e determinantes para a vulnerabilidade da pessoa idosa frente ao HIV/AIDS (Aguiar; Leal; Marques, 2020). Considerando que, isso dificulta o diálogo e debates referentes à temática e impede que o idoso possa sanar questionamento ou dúvidas, esta condição tende a representar também um empecilho para o incentivo de relações sexuais protegidas (Affeldt et al., 2015). O desenvolvimento de estratégias multidisciplinares contribuiu para a garantia da sobrevida e das condições de vida das pessoas que convivem com HIV/AIDS. Dessa forma, o aumento da sobrevida exigiu a necessidade de novas questões a serem discutidas, como por exemplo, a chance de tratamento e a necessidade de vínculos da rede social de apoio para o enfrentamento do preconceito e estigmas que ainda permanecem na sociedade (Palácio et al., 2012).

Nessa perspectiva, com o objetivo de atender as necessidades relacionadas à saúde do idoso com qualidade para cada nível assistencial, o Ministério da saúde criou estratégias para a organização e implantação de Redes Estaduais de assistência ao idoso. A implementação de serviços que possam referenciar as necessidades de cada grupo é de extrema importância para atingir a população idosa. No que tange ao tratamento do HIV/AIDS, o próprio Ministério da Saúde instituiu o centro de referência para IST's contando com uma equipe multiprofissional responsáveis pelas ações a serem garantidas a essas pessoas (Ferreira et al., 2014).

A presença do HIV/AIDS na vida da pessoa idosa tem um impacto prejudicial na qualidade de vida. Logo, o cuidado de si mesmo e como enfrentar a doença gera preocupações e também reflexão de como superar as consequências advindas junto com a sorologia positiva. Uma vez que, é preciso gerenciar de maneira positiva todo tratamento medicamentoso, o preconceito familiar e social, os aspectos psicológicos e ainda elaborar novas estratégias de saúde para alcançar um bem-estar. Assim, justifica-se a importância de compreender quais as estratégias de cuidado são utilizadas pela pessoa idosa que convive com o HIV/AIDS para que possam aprofundá-las e inseri-las nas redes de saúde como contribuinte para a saúde biopsicossocial e nas relações interpessoais.

Anteposto, esta pesquisa apresenta como objetivo conhecer as estratégias de cuidado à saúde desenvolvidas pela pessoa idosa em sua vivência com o HIV/AIDS.

\section{Metodologia}

Trata-se de um estudo exploratório, de caráter descritivo e com abordagem quanti-qualitativa. A pesquisa do tipo exploratória proporciona uma visão geral de um determinado fato, do tipo aproximativo (Gil, 2008). Já a abordagem qualitativa, conforme Silva e Menezes (2001, p.20), "considera que há uma relação dinâmica entre o mundo real e o sujeito, isto é, um vínculo indissociável entre o mundo objetivo e a subjetividade do sujeito que não pode ser traduzido em números". 
E a pesquisa quantitativa compreende na coleta sistemática de dados, com ênfase na objetividade e sua análise se dá normalmente por meio de procedimento estatístico. Assim, prontamente seremos conduzidos ao cenário do estudo.

Esta pesquisa foi desenvolvida em um Centro de Atenção e Apoio especializado de referência regional em atendimento médico, psicoterápico e social a pessoas que convivem com IST's e HIV/AIDS em um município do interior da Bahia. Refere-se à um recorte do projeto matriz intitulado "Envelhecer com HIV/AIDS: Memória e Representações Sociais de Idosos que convivem com o Vírus".

Participaram da pesquisa 26 pessoas idosas diagnosticadas com HIV/ADIS e que fazem tratamento no centro de referência da cidade. Assim, foi adotado como critério de inclusão no estudo: ter idade igual ou superior a 60 anos, de ambos os sexos, com diagnóstico de HIV/AIDS e estar cadastrado na unidade de referência no período da pesquisa. Nesse sentido, os critérios de exclusão restringiram a pessoas idosas com déficit cognitivo, que impossibilite a participação na pesquisa. Os instrumentos para coleta de dados utilizados foram: questionário com dados sociodemográficos e de saúde da pessoa idosa, roteiro semiestruturado para entrevista, todos construídos pelos pesquisadores. Utilizou-se também o Mini exame do Estado Mental (MEEM) na forma reduzida, para avaliar o estado mental (condições cognitivas) dos idosos, empregado para rastrear a presença ou não de comprometimento cognitivo, composto por 10 questões de certo e errado.

O MEEM completo é composto por duas seções que avaliam as funções cognitivas. Na primeira seção avalia a orientação, a memória e a atenção, totalizando 21 pontos. Já a segunda seção avalia a capacidade de nomeação, de obediência a um comando verbal e a um escrito e de cópia de um desenho complexo, no caso um polígono, totalizando nove pontos. O escore total é de 30 pontos e o ponto de corte é 23/24, sendo esta uma pontuação sugestiva de déficit cognitivo (Folstein \& Mchugh, 1975). O roteiro para entrevista semiestruturada foi empregado pelos próprios pesquisadores aos idosos, constituído por um roteiro de núcleos temáticos e questões abertas que contemplou aspectos relacionados ao objetivo do estudo.

Os dados quantitativos inicialmente foram inseridos em uma planilha do EXCEL e em seguida transportados para o Programa Estatístico SPSS versão 21.0, para realização da análise descritiva. Para análise dos dados qualitativos utilizou-se os recursos do Software Nvivo e após seleção das categorias utilizou a análise de conteúdo proposta por Bardin. Segundo Bardin (2016), o método de análise do conteúdo se organiza em três fases fundamentais: 1) pré-análise, 2) exploração do material e 3) tratamento dos resultados, inferência e interpretação.

A primeira fase, na pré análise houve uma leitura "flutuante" que permitiu a escolha dos documentos, formulação das hipóteses e os objetivos da pesquisa. Já a segunda fase, chamada de exploração do material ocorreu a definição das categorias e de acordo com o relato dos entrevistados a junção das falas correlativas. Por fim, na terceira e última fase, realizou-se o tratamento e interpretação dos resultados obtidos, o qual designou a análise e avanço da pesquisa.

Este estudo foi realizado atendendo os aspectos éticos pertinentes a pesquisas envolvendo seres humanos de acordo com as normas expressas na resolução 466/2012 do Conselho Nacional de Saúde tendo sido submetido à avaliação do Comitê de Ética em Pesquisa da Faculdade Independente do Nordeste, Parecer n 3.394.696.

\section{Resultados e discussão}

\section{Caracterização dos participantes do estudo}

Em relação aos dados sociodemográficos constatou-se um maior predomínio de idosos do sexo masculino $(69,23 \%)$, estado civil relativo a sem companheiro $(65,38 \%)$, com escolaridade $(84,62 \%)$ e renda financeira de até um salário mínimo $(69,235)$.

Quanto ao perfil das pessoas idosas do estudo houve uma predominância de idosos do sexo masculino no total de 18 homens e 8 mulheres, tais resultados são semelhantes aos demais estudos os quais apontam que os homens são os mais acometidos pela infecção. Em contrapartida, é observado também um crescimento significativo do número de casos entre as 
mulheres. Esse fato é baseado na vulnerabilidade feminina em que as mulheres são expostas ao vírus através de relações sexuais desprotegidas com o parceiro associado a impossibilidade de uma gravidez, uma vez que, essas mulheres já entraram na fase da menopausa. Em relação ao estado civil, o predomínio de idosos sem companheiros (17 idosos), corrobora com o risco dos idosos se infectarem pelas IST's, uma vez que, estão mais propensos a terem relações sexuais desprotegidas e sem parceiro fixo (Araldi et al., 2016).

Ademais, existe uma correlação entre os indicadores socioeconômicos e o aumento da incidência de HIV/AIDS, pessoas com baixa renda e escolaridade são as mais afetadas pela infecção. No entanto, o presente estudo vai em direção contrária a literatura onde 22 idosos da pesquisa referiram possuir escolaridade (84,62\%). Chamando atenção para este aspecto, é relevante entender que esse indicador gera de alguma maneira impacto na saúde do indivíduo, uma vez que, isso está atrelado ao acesso e a compreensão das informações transmitidas pelos profissionais. Nesse sentido, é um influenciador na adesão ao tratamento, pois o idoso pode compreender sobre medidas de prevenção, modo de uso das medicações e como prosseguir com o tratamento com maior clareza e facilidade, diferentemente de idosos sem escolaridade que podem apresentar dificuldades em ter conhecimento sobre as medidas de prevenção, formas de contágio e incapacidade de como seguir com o tratamento (Araldi et al., 2016).

Sabe-se que com a transição demográfica tem ocorrido cada vez mais incidentes de veiculação sexual em idosos, como por exemplo, o acometimento pelo HIV/AIDS e isso tem levantado discussão a nível nacional e internacional uma vez que esse aumento interfere negativamente na qualidade de vida dessas pessoas (Silva et al., 2018; Lopes et al., 2016).

Consta-se, que o envelhecimento populacional tem relação com as seguintes variáveis: a evolução da expectativa de vida, diminuição da taxa de natalidade e queda na taxa de mortalidade. Essas mudanças epidemiológicas permitiram um aumento quantitativo de idosos na sociedade (Ceccon, 2020). Nesse sentido, existe uma distinção entre o envelhecimento populacional e o individual, uma vez que, o crescimento da população idosa na sociedade está relacionado ao número de idosos crescentes de forma contínua e a redução na quantidade de jovens (Farias et al., 2019).

O envelhecimento sofre influências sociais, culturais e políticas em um âmbito coletivo, no entanto, é compreendido de maneira individualizada. Assim, é caracterizado como um evento intrínseco à condição biológica dos seres humanos, o qual sofrerá alterações biofísicas, psicológicas e/ou comportamentais (Chaves, 2017).

Após o diagnóstico positivo para o HIV/AIDS, múltiplos sentimentos envolvem o idoso. A primeira reação se remete a um choque por não acreditarem que foram infectados, seguidamente desencadeia a negação. Verifica-se que, a percepção que os idosos detêm sobre o HIV/AIDS é de uma "doença do outro", algo que nunca irá acontecer com eles. Posteriormente ao acometimento existe uma reflexão profunda sobre a vida de si e dos outros, e assim apresentam solidão, tristeza, vergonha, culpa, incredulidade, permitindo que o resultado gere um impacto maléfico sobre sua vida desejando muitas vezes a morte (Alves et al., 2020; Souza et al., 2016).

Nessa perspectiva, diante da descoberta da sorologia positiva para o HIV/AIDS os idosos enfrentam inúmeros desafios, dentre eles o preconceito da sociedade, familiares e amigos. Tendo em vista, o estigma enraizado no meio social e diante dessa situação o idoso procura desenvolver estratégias para lidar com a doença, essas estratégias funcionam como subsídios para aliviar o sofrimento e fortalecer a resistência no enfrentamento da infecção. Um importante mecanismo de defesa contra as acusações vindas do cenário social é o sigilo. O idoso após receber o resultado positivo para HIV/AIDS por medo de sofrer perseguições e ser excluído de um convívio familiar e coletivo mantém o diagnóstico privado, relatando exclusivamente para uma única pessoa, alguém próximo e confiável, como por exemplo, para o companheiro (a) e filho (Alves et al., 2020). 
$\mathrm{Na}$ avaliação das condições de saúde verificou-se que $(84,62 \%)$ dos idosos encontram-se na fase assintomática, enquanto apenas $(15,39 \%)$ dos idosos apresentavam complicações correlacionada a doença como anemia $(3,85 \%)$, baixo peso $(7,69 \%)$ e diarreia crônica $(3,85 \%)$.

É sabido que antes de surgir a TARV os indivíduos infectados pelo vírus do HIV/AIDS apresentavam maiores desordens nutricionais como a caquexia, ou seja, uma perda excessiva de peso, desnutrição e atrofia muscular, porém com a implementação do tratamento medicamentoso esse quadro mudou. Não obstante, após sua efetuação e seus inúmeros benéficos, sabe-se, que existem diversos desequilíbrios metabólicos correlacionados aos efeitos adversos da TARV e que resultam em uma instabilidade no organismo. Ademais, uma complicação característica das pessoas acometidas pelo vírus é a perda de peso e consequentemente um agravamento do estado funcional. Assim, é uma condição desencadeadora e que influencia para as infecções oportunistas (Silva, 2017; Souza, 2017).

Segundo Silva, et al., (2010) há evidências correlacionadas a um crescimento de síndrome metabólica em indivíduos contaminados pelo vírus HIV/AIDS e que utilizam a terapia antirretroviral, quando comparado a pessoas normais. Indo de encontro a esse aspecto, pode resultar em dislipidemia, resistência à insulina, diabetes Mellitus, entre outras (Gomes, 2016).

Outrossim, é que a diarreia se configura como uma complicação da infecção ou de infecções oportunistas que acometem o sistema digestivo. Assim, ela interfere na absorção dos nutrientes, a qual se torna um fator para sinais e sintomas de desnutrição, consequentemente distúrbios metabólicos (Liguori et al., 2017).

Desse modo, o que influencia negativamente na qualidade de vida dos idosos com HIV/AIDS é o fato da presença de doenças oportunistas, o que favorece maior sintomatologia e concomitantemente o uso de mais medicações. Assim, pode-se afirmar que os indivíduos que compõem a taxa de idosos sintomáticos apresentam qualidade de vida inferior à dos idosos assintomáticos.

\section{Estratégias de cuidado desenvolvidas pelos idosos para enfrentamento do HIV/AIDS}

As estratégias de cuidados desenvolvidas pelos participantes para o enfrentamento do HIV/AIDS serão apresentadas divididas em sete (7) subcategorias, sendo elas: Tratamento medicamentosos; Religiosidade; Prevenção Sexual; Atividade Física; Alimentação; Família, e Apoio Institucional, conforme segue:

\section{Tratamento medicamentoso}

A terapia antirretroviral (TARV) foi referida pelos idosos da pesquisa como uma estratégia de enfrentamento do HIV/AIDS, tendo em vista, os benefícios obtidos mediante ação farmacológica de proporcionar carga viral indetectável aos pacientes e como forma de sobrevivência e melhoria da qualidade de vida aos pacientes acometidos pelo vírus.

Olha, tomando meus remédios direito, me alimentando bem [...]. (E11)

Eu tomo medicamento no horário certo, não falho nenhum dia; me alimento bem, durmo bem, é isso. (E16)

Olha da minha saúde eu cuido assim, se é pra tomar os remédios eu tomo direitinho, sempre na saúde a gente gosta de alimentar as coisas direitinho. [...] (E23)

O Brasil deu um passo à frente na implantação de medidas governamentais e sociais no combate à epidemia da AIDS. Nos últimos tempos observou-se significativa diminuição nos níveis de mortalidade por AIDS no país, em virtude da inclusão do acesso gratuito à (TARV) e a oferta de testes diagnósticos disponíveis nas mais diversas redes de atenção à saúde. Essas combinações têm colaborado para a redução das internações hospitalares, diminuição das taxas de transmissão e 
concomitantemente para a qualidade de vida e o aumento da expectativa de vida das pessoas que convivem com a doença (Ribeiro et al., 2020).

As pessoas asseguram-se a adesão ao tratamento antirretroviral como uma saída de estratégia para melhor conduzir esse momento da doença, como uma forma de melhoria na qualidade de vida e uma esperança diante de um diagnóstico de uma patologia incurável, além de ser uma forma de autocuidado e adaptação a uma nova fase após o diagnóstico (Brandão et al., 2020). Além dessas medidas, a participação de idosos em grupos da terceira idade amplia os laços sociais e promove atividades de lazer, reduzindo o sentimento de abandono e exclusão a essa parcela da população.

Outro ponto positivo frente à política de prevenção ao HIV/AIDS, refere-se à introdução da PREP (profilaxia préexposição) e da PEP (profilaxia pós exposição) e é indicada pela Organização Mundial de Saúde as populações mais expostas a infecção como os homens que fazem sexo com homens, profissionais do sexo e transexuais. O uso da PREP é indicado para pessoas não infectadas pelo vírus do HIV que vão se expor ao ato de risco, por outro lado a PEP é utilizada em casos após pessoas terem tido um possível contato com o vírus em situações de violência sexual ou relações sexuais desprotegidas (Silva \& Cueto, 2018).

\section{Religiosidade}

Nessa categoria há relatos em que a religião e a crença em Deus representam uma estratégia de força para conviver com o HIV/AIDS, sendo utilizada pela pessoa idosa como importante estratégia de resiliência.

Normal.... Eu faço minha oração, depois da oração eu converso com as pessoas, com as pessoas certas, entendeu? $[\ldots](E 05)$

\section{[...] Oração, Deus na vida. (E 10)}

\section{[...] Eu vivo minha vida tranquila, graças a Deus. E graças a Deus eu me cuido [...] (E04)}

Nesse sentido, logo após o resultado positivo para a sorologia do HIV/AIDS, o idoso como qualquer outro indivíduo perpassa por um período de transição em sua vida decorrente dos estigmas e pelos novos hábitos de vida a serem aderidos. Assim, é sabido que um sentimento de luto envolve a pessoa infectada e procurando meios de ter um suporte, a religiosidade e espiritualidade são adotados. Não obstante, o coping religioso pode gerar conflitos, ou seja, interferir de maneira positiva ou negativa no enfrentamento do processo saúde e doença (Silva, 2018).

De forma a apresentar experiências positivas pelas pessoas que convivem com o vírus, a religiosidade e a fé em Deus fortalecem os idosos perante a doença, ajuda a ter as esperanças renovadas e encontrar um conforto espiritual que favorece o processo de aceitação da infecção, além de promover melhor adesão ao tratamento medicamentoso. Por outro lado, o indivíduo ao buscar explicações para o acometimento da doença, sente-se culpado e entende como um castigo de Deus por erros do passado e são condicionados a um julgamento religioso (Silva, 2018).

Segundo Reis e Menezes (2017) a prática da religiosidade e da espiritualidade é exercida pelos os idosos como um meio de favorecer e auxiliar no processo de envelhecimento saudável. Nesse contexto, sabe-se que as ações espirituais e religiosas promovem uma sensação de bem-estar ao indivíduo, pois elas provocam excitação neuroquímicas cerebrais as quais diminuem o estresse e ansiedade, como também aumentam a felicidade, as esperanças e tranquilidade.

Com isso, a execução da fé tem se mostrado como um método que encoraja o enfrentamento das diversidades advindas com o processo de envelhecimento. Logo, a fé em Deus é uma alternativa para os idosos à frente do adoecimento pela infecção do HIV/AIDS, a qual alivia o estresse psicológico e manter-se com um pensamento positivo diante do resultado pela infecção do vírus, permitindo contribuir com a saúde mental e física. 


\section{Prevenção sexual}

A prevenção sexual é relatada pelos idosos como uma forma de proteger os parceiros sorodiscordantes em razão do medo de infectar as pessoas com que se relacionam.

[...] quando eu saio e você sabe é bom ter uma namoradinha, previne, todo cuidado para não acontecer mais né? (E19)

[...] Eu não vou trair minha mulher, mas se chegar uma oportunidade eu não vou arriscar a vida de ninguém, porque eu não quero isso pra ninguém, entendeu? (E24)

\section{[...] Tem que ter cuidado pra não passar pra ninguém né? (E8)}

Percebe-se que a sociedade traz consigo, de geração em geração, o preconceito contra a pessoa idosa diante de sua vivência sexual. A coletividade baseada em costumes e crenças agregam tabus e ideias errôneas sobre o desejo sexual, e acreditam que não existe a prática do sexo entre pessoas idosas pela ausência da libido e rotulam os idosos como assexuais (Santos et al., 2017).

Contudo, diante do processo de envelhecimento, sabe-se que, ocorrem alterações fisiológicas, físicas e hormonais, como as quedas nas taxas de testosterona e estrogênio, que podem implicar em algumas alterações na libido ou na ereção. Entretanto, isso não é uma regra para extinguir a sexualidade, visto que, a sexualidade de um indivíduo não está restrita a penetração. O que é possível observar na pessoa idosa é que em decorrência dessas modificações pode haver uma redução erétil nos homens e uma diminuição na lubrificação vaginal na mulher (Silva, 2020).

Sob esta ótica, a sexualidade humana é considerada como uma função vital para a satisfação das necessidades básicas. Assim, a prática sexual envolve fatores biológicos, psicossociais e culturais, e promove bem-estar, prazer e elevação da autoestima do ser humano sendo, portanto, algo natural e que continua a ser possível na velhice. (Silva, 2020). Vale ressaltar que, além do sexo ser compreendido como uma prática física abrange sentimentos relevantes para a manutenção de um relacionamento e/ou satisfação física e mental. Assim sendo, o carinho, a troca de afeto, as expressões de amor, segurança e companheirismo, fazem parte do sexo ou em determinados momentos permitem e são considerados tão importantes quanto o ato sexual (Santos et al., 2017).

\section{Atividade física:}

Quanto a atividade física os idosos relataram que praticam exercícios constantemente em busca de um bem-estar físico e mental, conforme as falas a seguir:

Quando eu levanto, eu vou e escovo meus dentes, tomo meu café, faço exercício físico durante $1 \mathrm{~h},[. .$.$] mais tarde eu$ vou para caminhada de novo. (E 06)

Ah eu faço atividade física, caminhada, corrida... tô sempre no esporte de alguma forma, atividade física me ajudou bastante. Faço grupo de vez em quando, faço yoga. (E2)

Atividade física e meus remédios. (E22)

Percebe-se nesses depoimentos que entre os fatores que podem influenciar positivamente na qualidade de vida dos idosos com HIV/AIDS está a prática de atividade física. Assim, os idosos aprendem a gerenciar o exercício físico juntamente com o tratamento antirretroviral, ficando claro que houve mudança de paradigmas onde ter um diagnóstico de HIV/AIDS não é mais uma sentença de morte (Santana et al., 2018). 
Para Santos et al. (2021) a qualidade de vida depende de fatores cognitivos que na maioria das vezes estão reduzidos devido os fatores físicos, psicológicos e sociais gerando depressão e ansiedade. Vale ressaltar que, diante dos dados da presente pesquisa pode-se afirmar que os idosos que convivem com HIV/AIDS estão envelhecendo com uma excelente qualidade de vida, no qual a maioria teve o diagnóstico ainda na juventude e mantém um prognóstico positivo associado a adesão ao tratamento antirretroviral e a prática de exercício físico.

Desse modo, a assistência de saúde em especial da enfermagem prestada a esse público prioriza ações de autocuidado e da capacidade funcional voltada a atividades que estimulem a qualidade de vida dos idosos com abordagem multidisciplinar, além de enfatizar a esse público que eles podem e devem ser ativos independente da sua idade levando em consideração seu estado clínico geral (Santana et al., 2018; Santos et al., 2021).

\section{Alimentação}

A rotina alimentar é caracterizada como outro método de estratégia de cuidado, a qual visa a manutenção da qualidade de vida e um bom estado nutricional.

\section{Minha maneira é na alimentação. Como normal, como verdura, comidinha suave. (E09)}

Me cuido muito bem, alimentação na hora certa, me protejo ao sair com casaco. Faço meus exames $e$ acompanhamentos rotineiros. (E14)

Ah muito bem, alimentação na hora certa... (E03)

Em decorrência do aperfeiçoamento das tecnologias medicamentosas e a disponibilidade da TARV, verificou-se um aumento da sobrevida das pessoas acometidas pelo vírus do HIV. Assim, em detrimento dessa longevidade há uma preocupação com a manutenção da qualidade de vida. Nesse sentido, a adesão a uma alimentação adequada tornou-se um fator contribuinte é necessário para uma base nutricional eficaz, a qual auxilia no cuidado do bem-estar e também previne complicações ocasionadas pela própria terapia e pela doença.

Outro ponto que chama atenção nesse contexto é que, diferentemente do estudo apresentado por Silva (2010) e seus colaboradores, os resultados aqui apresentados evidenciam uma boa prática alimentar desenvolvida pelos pacientes, a qual também contesta com o que é abordado por grande parte da literatura. Nessa perspectiva, a alimentação adequada favorece a uma melhora no quadro clínico, decorrente do fortalecimento nutricional e do sistema imune ao se corrigir os déficits metabólicos.

Observa-se que os pacientes demonstram uma preocupação com a alimentação e em manter uma rotina alimentar, a fim de usufruir de boas condições de saúde e reduzir o risco de desnutrição e/ou complicações nutricionais. Desse modo, essa estratégia de autocuidado promove uma melhoria na qualidade de vida, além de aumentar o bom prognóstico da patologia.

\section{Família}

Os idosos relataram que o apoio familiar compreende uma maneira de amenizar os sofrimentos oriundos do diagnóstico positivo de HIV/AIDS.

A minha filha que cuida, porque eu mesmo não tenho cuidado nenhum. Alimentação eu não cuido não, se jogo mesmo (risos). [...] (E17)

Eu vivo muito bem [...] a minha família não teve preconceito nenhum, sou tratado como os outros, ou talvez até melhor. [...] (E06) 
A rede familiar constitui uma das mais importantes redes de apoio psicossocial, pois auxilia o indivíduo desde o momento do diagnóstico, no enfrentamento da nova condição de saúde e até a adesão ao tratamento antirretroviral.

A família é vista como a rede de apoio de maior impacto ao paciente, pois é possível perceber que a presença da AIDS na família funciona como um misto de sentimentos de medo, negação, tristeza, depressão, e que a falta do apoio da família faz com que esses sentimentos sejam acentuados, enquanto que com o apoio da rede familiar há o fortalecimento e motivação das pessoas que convivem com HV/AIDS (Brandão et al., 2020; Marcelino et al., 2019; Silva et al., 2015).

Conforme evidenciado nesse estudo, além das estratégias de cuidado desenvolvidas pelas pessoas idosas com HIV/AIDS, esses indivíduos reconhecem o apoio recebido pelos profissionais de saúde no processo de autocuidado.

\section{Apoio institucional}

O suporte das redes de apoio foi mencionado como estratégias que impulsionam a adesão do tratamento antirretroviral.

Depois de quatro anos mais ou menos, eu entrei em contato com eles e contei, uns aceitam, outros não, a família que eu considero é aqui, lá fora eu não considero família não. [...] (E01)

O apoio institucional é de suma importância no enfrentamento da doença e também na adesão ao tratamento, as informações, cuidados, apoio e diálogo das redes de apoio com os pacientes são vistas como mecanismos que impulsionam positivamente no enfrentamento do HIV/AIDS, pois corroboram para que os idosos se sintam acolhidos no processo da nova condição de saúde. Em face do estresse e das limitações por causa da soropositividade, o trabalho desenvolvido pela equipe multiprofissional é fundamental para um bom prognóstico. Assim, é possível afirmar que a postura adotada pelo profissional de saúde é determinante para adesão ou não do tratamento, e que tal aspecto pode facilitar ou dificultar o modo como o idoso irá enfrentar a doença. Portanto se torna indispensável o acolhimento, uma demonstração de cuidado e empatia ao próximo, repassando para a pessoa idosa segurança e confiança, que se tornará uma rede de apoio essencial (Thiengo et al., 2017).

\section{Considerações Finais}

Os resultados desta pesquisa desvelaram diferentes estratégias de cuidado à saúde desenvolvidas pela pessoa idosa em sua vivência com o HIV/AIDS, descritas como tratamento medicamentoso, religiosidade, prevenção sexual, atividade física, alimentação, família e apoio institucional. Enquanto alguns se asseguram ao tratamento antirretroviral, a prática de atividade física e a mudança de alimentação, outros alegam a fé e a religiosidade como subsídios para melhor conduzir a nova condição de saúde.

Ademais, os idosos ainda contam com apoio da equipe multidisciplinar (institucional) de saúde onde fazem o tratamento na unidade de referência para HIV/AIDS. Outro ponto importante de estratégia utilizada refere-se ao apoio do núcleo familiar onde o indivíduo encontra suporte psicossocial para lidar com o estigma e preconceitos que estão enraizados na sociedade até os tempos atuais.

Cabe ressaltar que, a equipe de saúde em especial a enfermagem atua de maneira a melhorar a qualidade de vida do idoso através de ações que possibilitem a troca de conhecimentos sobre a patologia e formas de transmissão, a importância do seguimento ao tratamento e consequências da não adesão ao tratamento antirretroviral, tendo assim, um olhar holístico para além do tratamento medicamentoso, implementando ações de cuidado nos diferentes aspectos biopsicossociais do paciente.

\section{Referências}

Affeldt, A. B., Silveira, M. F. \& Barcelos, R. S. (2015). Perfil de pessoas idosas vivendo com HIV/aids em Pelotas, sul do Brasil, 1998 a 2013. Epidemiol. Serv. Saúde, 24 (1), 79-86. 
Aguiar, R. B., Leal, M. C. C. \& Marques, A. P. O. (2020). Knowledgeand atitudes aboutsexuality in theelderlywith HIV. Ciência \& Saúde Coletiva, 25 (6), 2051-2062.

Alencar, R. A. \&Ciosak, S. I. (2015). O diagnóstico tardio e as vulnerabilidades dos idosos vivendo com HIV/AIDS. Rev. esc. enferm. USP,49 (2), $0229-0235$.

Alves, A. M., Venturi, A. F. A. \& Neto, J. M. F. A. (2020). A pessoa idosa e HIV/AIDS: descoberta, percepções e enfrentamento. Interciência\& Sociedade. 5 (1), 194- 209.

Araújo, L. F. et al. (2017). Concepções Psicossociais acerca do Conhecimento sobre a AIDS das Pessoas que vivem com o HIV. Rev. colomb. psicol., 26 (2), 219-230.

Araldi, L.M., et al. (2016). Pessoas idosas com o vírus da imunodeficiência humana: infecção, diagnóstico e convivência. Rev Min Enferm. 20 (4), 1-8.

Bardin, L. (2011). Análise de conteúdo. Edições 70.

Bastos, L. M. et al. (2018). Avaliação do nível de conhecimento em relação à Aids e sífilis por idosos do interior cearense, Brasil. Ciência \& Saúde Coletiva, $23(8), 2495-2502$.

Bittencourt, G. K. G. D. et al. (2015). Beliefsofolderadultsabouttheirvulnerabilityto HIV/Aids, for theconstructionofnursing diagnoses. RevBrasEnferm., 68 (4), 579-85.

Braga, J. M. S. R. \& Mariano, M. R. (2018). As representações das famílias frente ao processo do envelhecimento. Universidade da Integração Internacional da Lusofonia Afro-Brasileira.

Brandão, B. M. G. M., et al. (2020) . Convivendo com o HIV: estratégias de enfrentamento de idosos soropositivos. RevEscEnferm USP, 54.

Brito, A. M. D., Castilho, E. A. D. \&Szwarcwald, C. L. (2001). AIDS e infecção pelo HIV no Brasil: uma epidemia multifacetada. Rev. Soc. Bras. Med. Trop., 34 (2), 207-217.

Ceccon, R. F. et al. (2020). Envelhecimento e dependência no Brasil: características sociodemográficas e assistenciais de idosos e cuidadores. Ciência \& Saúde Coletiva, 26 (1), 17-26.

Chaves, R. N. (2017). Representações Sociais e Memória de idosos longevos sobre o processo de envelhecimento e a dependência funcional. Universidade Estadual do Sudoeste da Bahia.

Cruz, G. E. C. P. et al. (2020). Diagnóstico tardio do Vírus da Imunodeficiência Humana e da Síndrome da Imunodeficiência Adquirida em Idosos: protocolo scopingreview. EnfermeríaActual de Costa Rica, 38, 292-299.

Farias, M. F. R. N., Souza, P. H. V. \& Santos, V. E. S. (2019). O novo retrato demográfico do brasil: análise acerca do envelhecimento populacional e suas decorrências econômicas. Rev.Bras.de Direito e Gestão Pública, 7 (3), 01-11.

Ferreira, F. P. C. et al. (2014). Serviços de atenção ao idoso e estratégias de cuidado domiciliares e institucionais. Rev. bras. geriatr. gerontol., 17 (4), $911-926$

Folstein, M. F. \&Mchugh, P. R. A (1975). Practicalmethod for gradingthecognitivestateofpatients for theclinician. J. gsychiaf. Res. Pergamon Press. Printed in GreatBritain, 12, 189-198.

Gil, A. C. (2008). Métodos e técnicas de pesquisa social. Atlas.

Gomes, T. B. (2016). Perfil nutricional de pacientes HIV positivo do município de Apucarana (PR). Revista Saúde e Pesquisa, 9 (1), 83-92.

Lago, D. C. S. C., Rodrigues, C. X., Silva, J. B. A. \& Brasil, V. N. (2019). HIV/AIDS na terceira idade: desafios no processo de convivência familiar e social. In: VI Congresso Internacional de Envelhecimento Humano (CIEH), 1-9.

Liguori, M. M. B. C., Lisboa, R. C. \& Coutinho, V. F. (2017). Perfil nutricional de pacientes soropositivos em uso de antirretroviral. Nutrição Brasil, 16 (5), $344-50$.

Lopes, C. et al. (2016). HIV/AIDS na terceira idade prevenção e tratamento. Faculdade de São Paulo.

Marcelino, S. K. L. et al. (2019). Medidas estratégicas de assistência a pessoa idosa na prevenção contra HIV/AIDS. CIEH. 1-12.

Melo, L. A. et al. (2017). Fatores socioeconômicos, demográficos e regionais associados ao envelhecimento populacional. Rev. Bras. Geriatr. Gerontol. 20 (4), 494-50.

Nardelli, G. G., et al. (2017). Knowledgeaboutthehumanimmunodeficiencysyndromeamongelders in a unit for thecareoftheelderly. Rev Gaúcha Enferm,. 37, 19.

Nascimento, I. G. M. S. C. et al. (2021). Estigmas e preconceitos no viver-conviver com hiv/aids: um olhar sobre o estado da arte. REVISE - Revista Integrativa em Inovações Tecnológicas nas Ciências da Saúde, 1, 01-01.

Palácio, M. B. et al. (2012). O cuidado em HIV/AIDS e a atenção primária em saúde: possibilidades de integração da assistência. Psico, Porto Alegre, 43 (3), $350-36$.

Pedrosa, A \&Lebrego, A. M. (2016). Vulnerabilidade do idoso que vive com HIV/AIDS. Revista Kairós Gerontologia, 19 (4), 319-342. 
Research, Society and Development, v. 10, n. 17, e19101724219, 2021

(CC BY 4.0) | ISSN 2525-3409 | DOI: http://dx.doi.org/10.33448/rsd-v10i17.24219

Reis, L. A. \& Menezes, T. M. O. (2017). Religiosityandspirituality as resiliencestrategiesamonglong-living olderadults in theirdailylives. RevBrasEnferm, 70 (4), 761-6.

Ribeiro, L. C. S. et al. (2020). Diagnóstico tardio de infecção pelo Vírus da Imunodeficiência Humana e fatores associados. Rev. Latino-Am. Enfermagem, 28.

RiveroMarcotegui, M. et al. (2014). Diagnóstico tardio da infecção pelo vírus da imunodeficiência humana: oportunidades de diagnóstico perdidas. AnalesSis San Navarra, 37 (3), 329-338.

Santana, P. P. C. et al. (2018). Fatores que interferem na qualidade de vida de idosos com HIV/AIDS: uma revisão integrativa. Cogitare Enfermagem, 23 (4), 59117.

Santos, A. A. B. S. et al. (2021). Qualidade de vida de idosos vivendo com HIV/AIDS: revisão integrativa. Revista de Enfermagem. 14 (14), 96-108.

Santos, M.C. et al. (2017). Percepções e vivências de idosos sobre sua sexualidade. Almana multidisciplinar de pesquisa, 1 (1), 25-36.

Silva, A. G. S. et al. (2018). Revisão integrativa da literatura: assistência de enfermagem a pessoa idosa com HIV. RevBrasEnferm. 71 (2), 884-92.

Silva, A. L. B. (2018). A Influência das Crenças Religiosas e Espiritualidade na Adesão ao Tratamento de Pessoas Vivendo com HIV/Aids. Universidade Federal do Estado do Rio de Janeiro.

Silva, A. O., Loreto., M. D. S. \& Mafra, S.C.T. (2017). HIV na terceira idade: repercussões nos domínios da vida e funcionamento familiar. Rev. Em pauta. 15 (39), $129-154$.

Silva, E. F. R. et al. (2010). Estado nutricional, clínico e padrão alimentar de pessoas vivendo com HIV/Aids em assistência ambulatorial no município de São Paulo. RevBrasEpidemiol, 13 (4), 677-88.

Silva, E. L. \& Menezes, E. M. (2001). Metodologia da pesquisa e elaboração de dissertação. rev. atual. 121.

Silva, E. M. (2020). Sexualidade na velhice: discurso sobre o aumento dos casos de hiv/aids na população idosa. Universidade Federal da Paraíba.

Silva, L. C. D. et al. (2015). Impacto psicossocial do diagnóstico de HIV/Aids em idosos atendidos em um serviço público de saúde. Rev.Bras. Geriatr. Gerontol. 18 (4), 821-833.

Silva, S. R. A. (2017). Estado nutricional e fatores associados em pessoas idosas com HIV/AIDS assistidas nas unidades de referência do Recife. Universidade Federal de Pernambuco.

Sousa, L. R. M., et al. (2019). Representações sociais do HIV/Aids por idosos e a interface com a prevenção. RevBrasEnferm., 72 (5), $1192-9$.

Souza, C. N, et al. (2017). Perfil nutricional de pacientes HIV/AIDS hospitalizados. Multitemaas, 23 (53), 159-181.

Souza, M. D. D. et al. (2016). Conhecimento dos idosos da estratégia saúde da família em relação ao HIV/AIDS. Revenferm UFPE online. 10 (11), $4036-45$.

Thiengo, P. C. S., Gomes, A. M. T. \& Oliveira, D. C. (2017). As representações do cuidado voltado à pessoa que vive com HIV/AIDS para a equipe de saúde. Revista Enfermagem Atual. 82, 41-47. 